\title{
A Cosmic Census of Radio Pulsars with the SKA
}

\section{E. F. Keane ${ }^{* 1,2,3}$, B. Bhattacharyya ${ }^{4,5}$, M. Kramer ${ }^{6,4}$, B. W. Stappers ${ }^{4}$, S. D. Bates ${ }^{7}$, ${\text { M. } \text { Burgay }^{8} \text {, S. Chatterjee }}^{9}$, D. J. Champion ${ }^{6}$, R. P. Eatough ${ }^{6}$, J. W. T. Hessels ${ }^{10,11}$, G. Janssen ${ }^{10}$, K. J. Lee ${ }^{12,6}$, J. van Leeuwen ${ }^{10,11}$, J. Margueron ${ }^{13}$, M. Oertel ${ }^{14}$, A. Possenti ${ }^{8}$, S. Ransom ${ }^{15}$, G. Theureau ${ }^{16}$ \& P. Torne ${ }^{6}$}

${ }^{1}$ Swinburne University of Technology, Australia; ${ }^{2}$ ARC Centre of Excellence for All-Sky Astrophysics (CAASTRO); ${ }^{3}$ E-mail: ekeane@swin. edu . au; ${ }^{4}$ University of Manchester, $U K ;{ }^{5}$ E-mail: bhaswati . bhattacharyya@manchester.ac.uk; ${ }^{6}$ MPIfR Germany; 7 NRAO Green Bank, USA; ${ }^{8}$ INAF-Osservatorio Astronomico di Cagliari, Italy; ${ }^{9}$ Cornell University, USA; ${ }^{10}$ Netherlands Institute for Radio Astronomy (ASTRON), The Netherlands; 11 University of Amsterdam, The Netherlands; ${ }^{12}$ Peking University, P.R.China; ${ }^{13}$ Institut de Physique Nucléaire de Lyon, CNRS, France; ${ }^{14}$ Paris Observatory, France; ${ }^{15}$ NRAO Charlottesville, USA; ${ }^{16}$ CNRS-Université d'Orléans, France.

The SKA will make ground breaking discoveries in pulsar science. The wide field-of-view, high sensitivity, multi-beaming and sub-arraying capabilities, coupled with advanced pulsar search backends, will result in the discovery of a large population of pulsars and new, high-quality data on select sources from the known pulsar population. This chapter outlines surveys for new pulsars, as well as the necessary follow-up timing observations, which will enable all of the SKA's headline pulsar science goals (tests of General Relativity with pulsar binary systems, investigating black hole theorems with pulsar-black hole binaries, and direct detection of gravitational waves in a pulsar timing array). SKA Milky Way surveys at multiple frequencies will increase the number of known pulsars by more than an order of magnitude. SKA2 will potentially find all of the Galactic radio-emitting pulsars in the SKA sky which are beamed in our direction. This will give a clear picture of the birth properties of pulsars and of the gravitational potential, magnetic field structure and interstellar matter content of the Galaxy. Targeted searches will enable detection of the most exotic systems, such as the $\sim 1000$ pulsars we infer to be closely orbiting Sgr A*, the supermassive black hole in the Galactic Centre. In addition to Galactic pulsars, the SKA will detect pulsars from local group galaxies, which can be used as probes of the local intergalactic medium. All of the discoveries will require regular re-observations for a few months in order to derive their spin characteristics and establish the particular science questions they can be used to address. For efficiency, live searches will be performed, using sub-arraying and dynamic scheduling to time pulsars as soon as they are discovered, while simultaneously continuing survey observations. The large projected number of discoveries suggests that we will uncover currently unknown rare systems that can be exploited to push the boundaries of our understanding of astrophysics and provide tools for testing physics, as has been done by the pulsar community in the past.

Advancing Astrophysics with the Square Kilometre Array

June 8-13, 2014

Giardini Naxos, Italy

* Speaker. 


\section{Introduction}

The SKA will be a discovery machine. Apart from delivering transformational science based on the expected huge increase in pulsar timing precision, the SKA's high sensitivity, wide field of view (FoV), and frequency coverage will allow us to explore the variable radio sky in an unprecedented way. This will lead to the discovery of previously unknown types of sources and enable us to probe a wide range of explosive and dynamic events. Eventually, it will lead to a full census of detectable radio pulsars in the Milky Way and beyond. Among the new sources will be fast, spin-stable millisecond pulsars (MSPs) whose period distribution reflects the equation of state of nuclear matter and some of which will serve as detectors of nano-Hz gravitational waves (GWs). Relativistic binary pulsars, particularly those with orbital periods of a few hours or less, will allow strong-field tests of General Relativity and other theories of gravity. The discovered pulsars will also be superb probes for an enhanced understanding of the Milky Way, its structure and its constituents, including magnetic fields, the free electron distribution etc. Other applications across a wide range of physics and astrophysics topics are described in the accompanying chapters.

The first step toward these unique achievements will be enabled by Phase I of the SKA (referred to as SKA1 hereafter). Apart from being a transformational telescope in its own right, the first science phase of this unique telescope will set the scene for the experiments to be conducted with the full array. However, it is important to realize that for searching, SKA1 is not only a simple stepping stone towards SKA2. Due to limitations in processing power, it is unlikely that the full area of the completed SKA can be utilized for a blind, large-scale survey for some time to come. Hence, SKA1, with a highly concentrated core, represents a significant fraction of the collecting area usable for surveys with SKA2, and substantial achievements can be made in pulsar searching in the early science phase.

When considering the specifications for SKA1, we point out that, in general, a loss in sensitivity (i.e. collecting area) cannot be simply compensated by longer integration times. In the best case, a reduction in collecting area that can be phased up coherently may require a significant increase in computing power (both for beam-forming, and processing). These costs are typically prohibitive, e.g. as illustrated by Figure 1 a 30\% loss in raw sensitivity, if compensated for by doubling the observing time, results in a ten-fold increase in computation in order to find the same pulsars. Rephrased, this shows that as SKA1 approaches the optimal combination of dishes in the core region, pulsar searching becomes ever more efficient. In the worst case, a loss in sensitivity means a degradation in the science that is possible.

\subsection{Pulsar Searching Basics}

The pulsar signal is periodic with known periods covering nearly four orders of magnitude, i.e. $1 \mathrm{~ms}$ to $10 \mathrm{~s}$. The pulse duty cycle ranges, typically, from less than one degree in rotational phase to the full pulse period. Hence, the discovery of pulsars requires a search of the radio sky in dispersion measure, pulse period and pulse duty cycle. For binary pulsars, a search for acceleration is also required. The standard search technique is consequently computationally expensive and it involves the Fourier-transform of correspondingly prepared time-series where the search in duty cycle uses a technique known as harmonic summing. Apart from these data processing requirements, forming beams for a sufficiently large FoV to enable reasonable survey speed, is computationally 


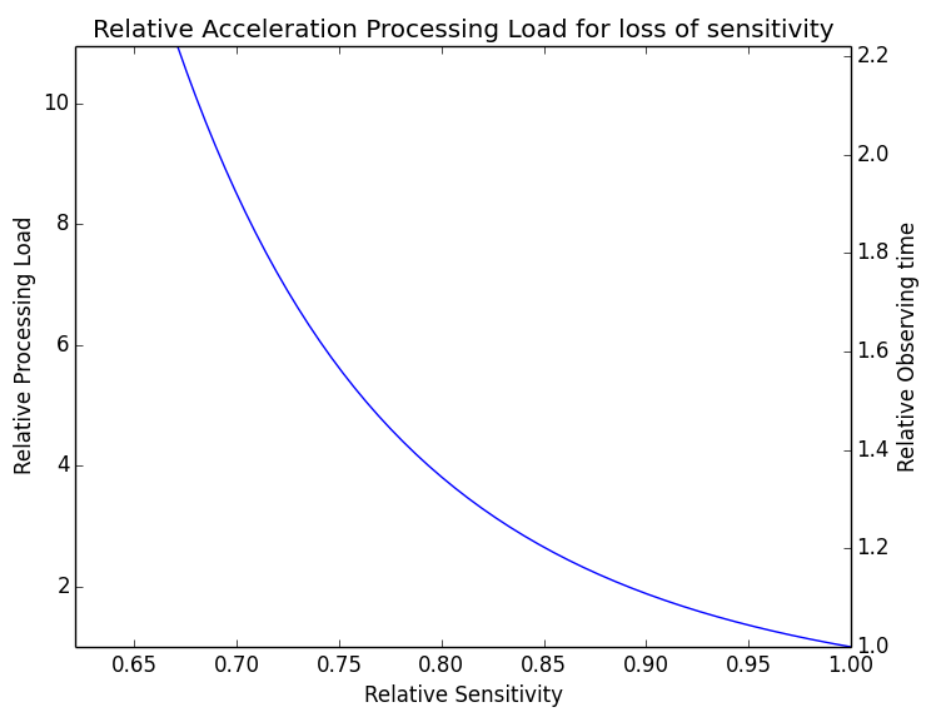

Figure 1: The 'cost', in terms of the increased observing time and (most significantly) computer processing required to find accelerated binary systems, in the event of a reduction in sensitivity.

challenging in the signal processing chain, in particular if the array configuration is not sufficiently compact. In order to ease the computational requirements, we have explored different observing strategies for the search for pulsars, depending on sky (i.e. Galactic) location, frequency (and hence receiver technology), and observing modes.

\subsection{Acceleration searches}

Arguably, the most interesting systems to be found will be highly accelerated. The SKA promises an advance over existing telescopes in the particular ability to allow for short integration times due to its large sensitivity. That means that only a small fraction of the orbit is sampled, so that the pulse frequency may not change significantly due to varying Doppler shifts. In contrast, reduced sensitivities imply longer integration times, which mean more Doppler-smearing that needs to be compensated for (if possible at all) by computational means. In the best case, one can assume constant acceleration. Even in the simplest acceleration search, the computing power needed scales with the cube of the observing time. In other words, a 10\%-reduction in sensitivity implies a required increase in computing efforts by a factor of two in order to find the same systems (see Figure 1). In practice, the penalty is much higher, for two reasons. Firstly, the previous assumption of constant acceleration will be wrong for the most compact systems when the integration time becomes a significant fraction of the orbital period (depending also on the unknown eccentricity of the system). Secondly, even if antennae at longer baselines could be included coherently in effective collecting area, it would require an increased amount of beam forming.

The impact on the population so-lost is difficult to quantify as the result depends not only on the (unknown) luminosity of the sources, but also on the orbital parameters. Population syntheses are used to infer information (see $\S 3$ ), but we can be certain that there are more compact orbits than the Double Pulsar $\left(P_{\mathrm{b}}=147 \mathrm{~min}\right)$ or the shortest pulsar binaries known $\left(P_{\mathrm{b}}=92 \mathrm{~min}\right)$. It 
is notable that the Double Pulsar was not detected in the regular pointings of the Parkes Multibeam Pulsar Survey ( $t_{\text {int }}=35 \mathrm{~min}$ ) because of Doppler-smearing. Only in the 10-times shorter integrations of the "PH"-survey, was it finally discovered. It is conceivable that most of the strong (mildly-accelerated) sources are discovered with the currently available sensitivity and computer resources. This would imply that the remaining sources are either relatively weak or very highly accelerated. In other words a loss in SKA sensitivity (the largest that we can ever expect to achieve) would suggest a certain (and final) blindness to the most exciting systems.

SKA radio pulsar surveys will produce a large volume of prospective candidates, the majority of which will be forms of noise. Typically, such large numbers of candidates need to be visually inspected in order to determine if they are real pulsars. This process can be labour intensive and delay the candidate confirmation. In order to process pulsar searching candidates in a real time fashion, computer software to perform candidate ranking or classifying is demanded. At present, software using machine learning (Eatough et al. 2010; Lyon et al. 2013; Zhu et al. 2014) or statistical classifiers (Lee et al. 2013; Morello et al. 2014) can increase the identification rate by a factor of about 50 to 1000 . This will significantly improve the efficiency of pulsar searches with SKA1 and SKA2, allowing for fast identification and confirmation of the candidates.

\subsection{Structure of this Chapter}

The remainder of this chapter is structured as follows: In $\S 2.1$ we describe the large parameter space probed in pulsar searches. Surveys with SKA-LOW and SKA-MID are described in $\S 2.2$ and $\S 2.3$, respectively. $\S 3$ describes the results of population syntheses, and the results of simulations of the survey yields, and evaluates different search strategies. In addition to the 'blind' surveys we describe targeted searches in $\S 4$. We review, in $\S 5$, the wide range of anticipated discoveries and their scientific importance. Finally, in $\S 6$ we discuss the important issue of the follow-up timing strategies of the newly discovered pulsars which are needed to maximize the scientific yield.

\section{Pulsar searches with the SKA}

\subsection{Parameter-space for search}

As described above, and elsewhere in this book, one of the key aims of the SKA, in both Phase 1 and with SKA2, is to discover as many pulsars as possible. The pulsars of interest for the different astrophysical goals may be located anywhere within, or even beyond, our Galaxy and so it is necessary to perform a "blind" survey of the entire sky visible from the sites in Australia and South Africa. To survey this area efficiently we need to achieve the maximum possible survey speed and this requires excellent sensitivity combined with a wide-FoV (Smits et al. 2009). The high time resolution required to discover pulsars means that it is not effective to search for them in the images that are the traditional data product of interferometers like the SKA. Instead we have to form the coherent sum of as many dishes/stations as possible to give us the required sensitivity. However, as the dishes are sparsely distributed, the FoV shrinks as we add more dishes to improve our sensitivity. This can be overcome by forming more beams, but that comes with a computing cost associated with forming and processing those beams. Depending on the exact configuration of the all-sky pulsar survey to be undertaken with SKA1 between 1500 and 2200 tied-array beams are 
required for SKA1-MID and 500 tied-array beams are required for SKA1-LOW. With SKA2 at least 10,000 beams will be required, which will allow more dishes to be included in the array, improving sensitivity, but while also increasing the FoV (this increased 'survey speed' also allowing longer integrations).

The radio emission from pulsars is dispersed, before it arrives at Earth, by the free electrons along the line of sight to the pulsar. This causes a frequency-dependent delay which needs to be removed in order to recover the pulsed signal. Unfortunately, the degree of dispersion cannot be predicted and so a search over a range of so-called dispersion measures is required. The maximum dispersion measure is of course highly dependent on where the pulsar is located, and with the majority of pulsars located in the Galactic plane, where the dispersion can reach its highest values, it is necessary to search over a wide range of dispersion measures. This is very important for the SKA where the sensitivity is high enough to allow us to discover the most distant sources. With SKA1 it is proposed that the search be possible out to dispersion measures of 3000 DM units; with the proposed search parameters - $\mid$ acc $\mid<350 \mathrm{~m} / \mathrm{s} / \mathrm{s}, 2048$ beams and harmonic folding - the computaional requirement is at least 7 Petaflop/s. However, with SKA2 and/or higher frequency observations it should be possible to search out to dispersion measures of 10,000 DM units and beyond.

To search for the systems critical to the key gravity studies, such as the pulsar-black hole (PSR$\mathrm{BH}$ ) and double neutron star (DNS) systems, requires that one is able to at least partially correct the modulation of the pulse frequency caused by its motion in the binary - usually via acceleration searches. The orbital acceleration that might be observed in compact PSR-BH binaries could be an order of magnitude larger than that seen in the most relativistic DNS systems (see $\S 5.1$ ). For the proposed integration time of SKA1 (10 min, see $\S 2.3$ ) the aim is to search for accelerations $>1000 \mathrm{~m} / \mathrm{s} / \mathrm{s}$; indeed using fluctuation frequency domain methods this will be readily achievable for spin periods greater than $>16 \mathrm{~ms}$ (Lorimer \& Kramer 2005). In highly eccentric PSR-BH systems, the acceleration can be larger by factors of a few near periastron. While at the moment acceleration searches (in both the time and frequency domain) over such ranges and with uniform spin frequency sensitivity are computationally prohibitive, for SKA this should be the goal.

\subsection{An all-sky pulsar survey with SKA-LOW}

All-sky pulsar searches are often most efficiently done using low radio observing frequencies $(100-600 \mathrm{MHz})$. Such surveys benefit from the typically steep spectra of pulsars $\left(S \propto v^{-1.6}\right.$; Bates et al. 2013), as well as the naturally larger FoV of the telescope (FoV scales as $v^{-2}$ for a fixed aperture size or baseline length). Interstellar propagation effects, most notably dispersive delay, multi-path propagation due to scattering, and sky temperature, are the main limitations at low frequencies. Dispersive delay, which scales as $v^{-2}$, is a correctable effect however, as long as the channel bandwidth is sufficiently narrow that intra-channel smearing of the pulse is minimal compared with the sampling time. Scattering, which scales as $\sim v^{-4}$, is not practically correctable in a blind survey and is a major limitation for finding distant pulsars in the Galactic plane, especially at short rotational periods. Sky temperature scales as $v^{-2.6}$, but is only $\sim 35 \mathrm{~K}$ (at $400 \mathrm{MHz}$ ) for high Galactic latitudes. For these reasons low-frequency pulsar surveys are the preferred option for Galactic latitudes beyond $|b| \approx 5$ degrees. 
Of the $\sim 2300$ known pulsars: there are 766 pulsars with $|b|>5$ degrees; of the 172 Galactic Fields MSPs $(\mathrm{P}<30 \mathrm{~ms})$ : 113, i.e. two thirds are at $|b|>5$ degrees (Manchester et al. 2005). Furthermore, population synthesis of the entire Galactic pulsar population indicates that if SKA1LOW and SKA1-MID are used in a complementary way to survey the entire SKA-visible sky, then the maximum yield of pulsar discoveries can be achieved (assuming a constant on-sky survey time) if SKA1-LOW surveys at Galactic latitude $|b|>5$ degrees and SKA1-MID surveys the Galactic plane.

There are currently three major, ongoing low-frequency pulsar surveys: (i) the Arecibo 327MHz Drift Survey ${ }^{1}$, operating from $300-350 \mathrm{MHz}$; (ii) the Green Bank Northern Celestial Cap Survey $^{2}$, which in a certain sense is a continuation of the Green Bank Drift-Scan Survey ${ }^{3}$ as well as the GBT350 Galactic Plane survey, and is operating from $300-400 \mathrm{MHz}$; and (iii) the LOFAR Tied-Array All-Sky Survey ${ }^{4}$, which is operating from $119-151 \mathrm{MHz}$ and is very similar in approach to how SKA-LOW will survey for pulsars and fast transients. Together, these low-frequency GBT, Arecibo and LOFAR surveys have discovered 176 pulsars in the last decade. The GBT surveys have used scan lengths of $\sim 2$ minutes and reached a minimum flux density $S_{\text {min }}^{350} \approx 1$ mJy. Each pointing covers 0.25 sq. degrees. The Arecibo survey provides only 40-s in-beam time, and reaches $S_{\min }^{350} \approx 0.5 \mathrm{mJy}$. Each pointing covers 0.05 sq. degrees. LOFAR's much larger FoV which is enabled by the use of 219 simultaneous tied-array beams to cover 9 sq. degrees of sky allows 1-hr integrations, which reach $S_{\min }^{150} \approx 1 \mathrm{mJy}$.

SKA1-LOW will provide an enormous leap in sensitivity and survey efficiency compared with these ongoing surveys. An all-sky survey using just the 600 -m (radius) core of SKA1-LOW along with 10 -min dwell time and 500 tied-array beams will reach $S_{\min }^{350} \approx 0.05 \mathrm{mJy}$ (ten times deeper than any ongoing survey) and will cover $\sim 1$ sq. degrees per pointing. We also note that the instantaneous sensitivity of the $600-\mathrm{m}$ SKA1-LOW core at $350 \mathrm{MHz}$ will be a factor of a few greater than Arecibo. Instantaneous sensitivity is the important factor for detecting weak individual pulses, like those from the intermittent radio pulsars and the fast radio bursts (see the Chapter on "Fast Transients at Cosmological Distances", Macquart et al. 2015, for a discussion of this).

We emphasize that a cosmic census of radio-emitting neutron stars requires the complementarity provided by SKA-LOW and SKA-MID (here, to clarify, we are referring to both SKA1 and SKA2). For the reasons outlined above (primarily scattering and $T_{\text {sky }}$ ), SKA-MID will be the main tool for discovering distant pulsars in the Galactic plane, whereas SKA-LOW can both go deeper and survey faster at higher Galactic latitudes. Together, these two surveys will characterize the spectra and distribution of Galactic radio pulsars in beautiful detail. They will both discover exotic individual systems which can be used to test theories of dense matter and gravity. For example the GBT Drift-Scan survey found PSR J0337+1715, a unique millisecond pulsar in a stellar triple system, which promises a very strong constraint on deviations from the Strong Equivalence Principle of General Relativity (Ransom et al. 2014).

\footnotetext{
${ }^{1}$ http://www.naic.edu/ deneva/drift-search/

${ }^{2} \mathrm{http}: / /$ arcc.phys.utb.edu/gbncc/

${ }^{3}$ http://astro.phys.wvu.edu/GBTdrift350/

${ }^{4} \mathrm{http}: / /$ www.astron.nl/lotaas/
} 


\subsection{Composite survey with SKA1-LOW and SKA1-MID}

To consider the usefulness of surveying with SKA1-LOW, we first compare the number and type of pulsars it will find with those found by SKA1-MID. We have shown with the help of simulations (see the next section for details) that all-sky surveys with SKA1-MID find a larger number of normal pulsars and MSPs than SKA1-LOW. However, with SKA1-LOW we will find many additional pulsars to those found with SKA1-MID, thereby the two greatly complement each other. While young pulsars (ages of $10^{3}-10^{7} \mathrm{yr}$ ) are strongly confined to their birth places in the Galactic plane (though sources at $\lesssim 1 \mathrm{kpc}$ will still appear to be isotropic), the much older MSPs (ages of $\gtrsim 10^{8} \mathrm{yr}$ ) will appear across the sky. This means that an all-sky pulsar survey is critical for finding the best pulsar clocks for use in direct GW detection and other fundamental physics experiments. Conversely, a deep Galactic plane search is needed to find the DNS binaries or the PSR-BH binaries that will provide the most stringent tests of General Relativity.

To carry out a large area survey with SKA1-LOW would require that a beamformer and a pulsar search backend be built. In the present scenario we have considered that we would use the collecting area of SKA1-LOW out to a radius of $700 \mathrm{~m}$ to include a total of 500 stations. Although this is a larger radius than used for SKA1-MID the significantly lower observing frequency means that the beam size is about 7 times larger for SKA1-LOW and so to achieve the same survey speed fewer beams would be required. We consider here a scenario where we preserve the total number of beams defined in the baseline design for the SKA1-MID beamformer of about 2048 beams and we split them across both telescopes with SKA1-LOW having 500 beams and SKA1-MID having 1500 beams. As the pulsar survey processing cost scales approximately linearly with the number of beams this transferring of the beams from SKA1-MID to SKA1-LOW is almost cost neutral. We also note that in this scenario the decrease in the area to be surveyed with SKA1-MID would allow for a smaller tied-array beam size. If one changes the integration times for SKA1-LOW to (say) $1800 \mathrm{~s}$ and SKA1-MID to $2600 \mathrm{~s}$ respectively, the relative yield will be different from the results obtained with a fixed integration time of $600 \mathrm{~s}$ for both telescopes. This optimization can be performed later, when further results from the ongoing surveys with Parkes, LOFAR and the GBT are available, enabling an informed judgment. It is clear, however, that SKA1-LOW should be equipped with searching capabilities.

\section{Simulations}

\subsection{SKA1 Simulations}

To determine how to achieve the maximal possible number of pulsar discoveries with SKA1 we have performed a number of simulations which take into account all of the potentially available resources. We then consider how this might project forward to SKA2. Our simulations presented here are based on the PSRPOP.py code (Bates et al. 2014) but are consistent with those obtained with the code used in van Leeuwen \& Stappers (2010) for LOFAR. The simulations only considered SKA1-LOW and SKA1-MID, as large area surveys with SKA1-SUR require integration times that are too long to achieve useful sensitivity and, therefore, require presently unachievable computational requirements to reveal the desired binary systems. 

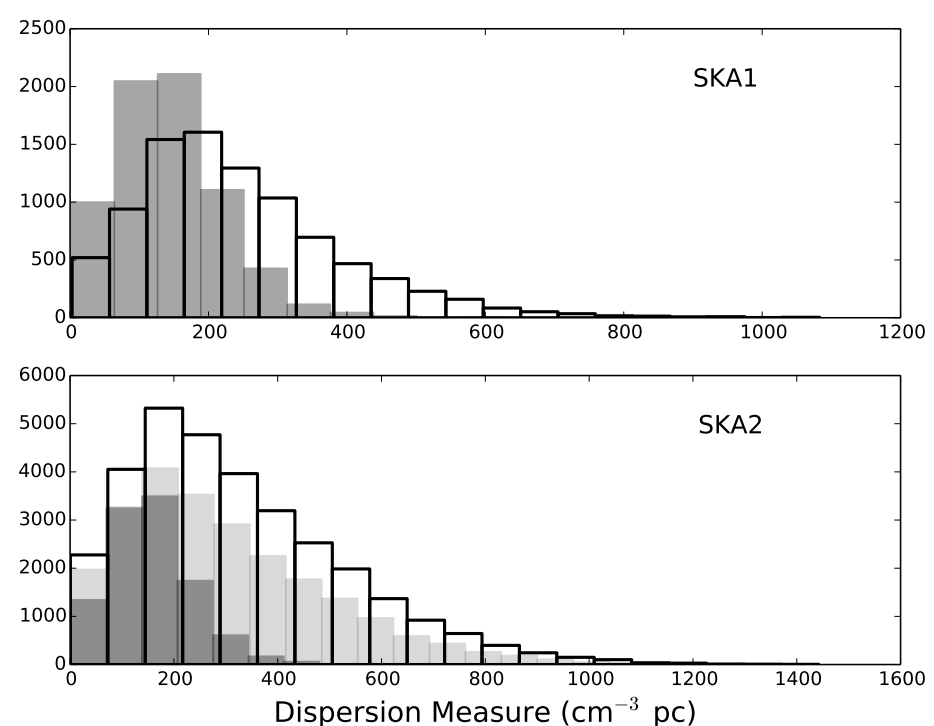

Figure 2: Histograms showing the pulsar search performance of the LOW and MID telescopes in both phases of the SKA. In SKA Phase 1 one can see how LOW (Dark bars) performs better at low dispersion measures due to the raw sensitivity but MID (clear bars) reaches deeper into the Galaxy. With the full SKA we consider two options for MID(DISH) of a five- and ten-fold improvement in sensitivity, light grey and clear bars respectively and also an improved LOW (dark bars). As with SKA1, LOW finds a different set of pulsars compared to MID.

We first considered the optimal frequency for pulsar searching with SKA1-LOW by performing simulations across the entire expected available band from $100-450 \mathrm{MHz}$ assuming sensitivity parameters as described in the baseline design. We find that, for a $100-\mathrm{MHz}$ bandwidth, the optimum central frequency is $250 \mathrm{MHz}$, where this is a compromise between pulsar spectral index, sky temperature and effective collecting area of the log-dipole antennas. Multiple simulations were then performed for all-sky surveys with SKA1-LOW and SKA1-MID assuming a fixed integration time of $600 \mathrm{~s}$ per pointing as described in the baseline design. These show that SKA1-MID would detect about 9000 normal pulsars and about 1400 MSPs while the numbers for SKA1-LOW are about 7000 normal pulsars and about 900 MSPs. It appears, therefore, that SKA1-LOW is less competitive, but it is important to remember that SKA1-LOW sees a smaller fraction of the sky and if one looks at the number of pulsars discovered as a function of dispersion measure, that SKA1LOW finds more pulsars at low dispersion measure. This clearly shows that the lower frequencies observed with SKA1-LOW mean that the dispersion smearing and the scattering in the ISM along lines of sight to and through the Galactic plane reduce the number of pulsars that can be detected there, while the superior collecting area of SKA1-LOW means that it is able to find more pulsars nearby.

The results of these simulations indicate that a composite survey, where the complementary regions are covered by the two telescopes, provides the best combination to maximise the number of pulsars that can be found with SKA1. We therefore performed composite survey simulations which used both telescopes to survey different regions to maximise the pulsar yield and we found that an optimal survey strategy would be to search with SKA1-MID up to Galactic latitudes of about \pm 10 degrees and the region of the sky in the North where SKA1-LOW cannot reach. SKA1LOW would then be used to survey the rest of the sky down to a Galactic latitude of \pm 5 degrees. The reason for the overlap in this region is two-fold: firstly it allows effective cross-calibration 

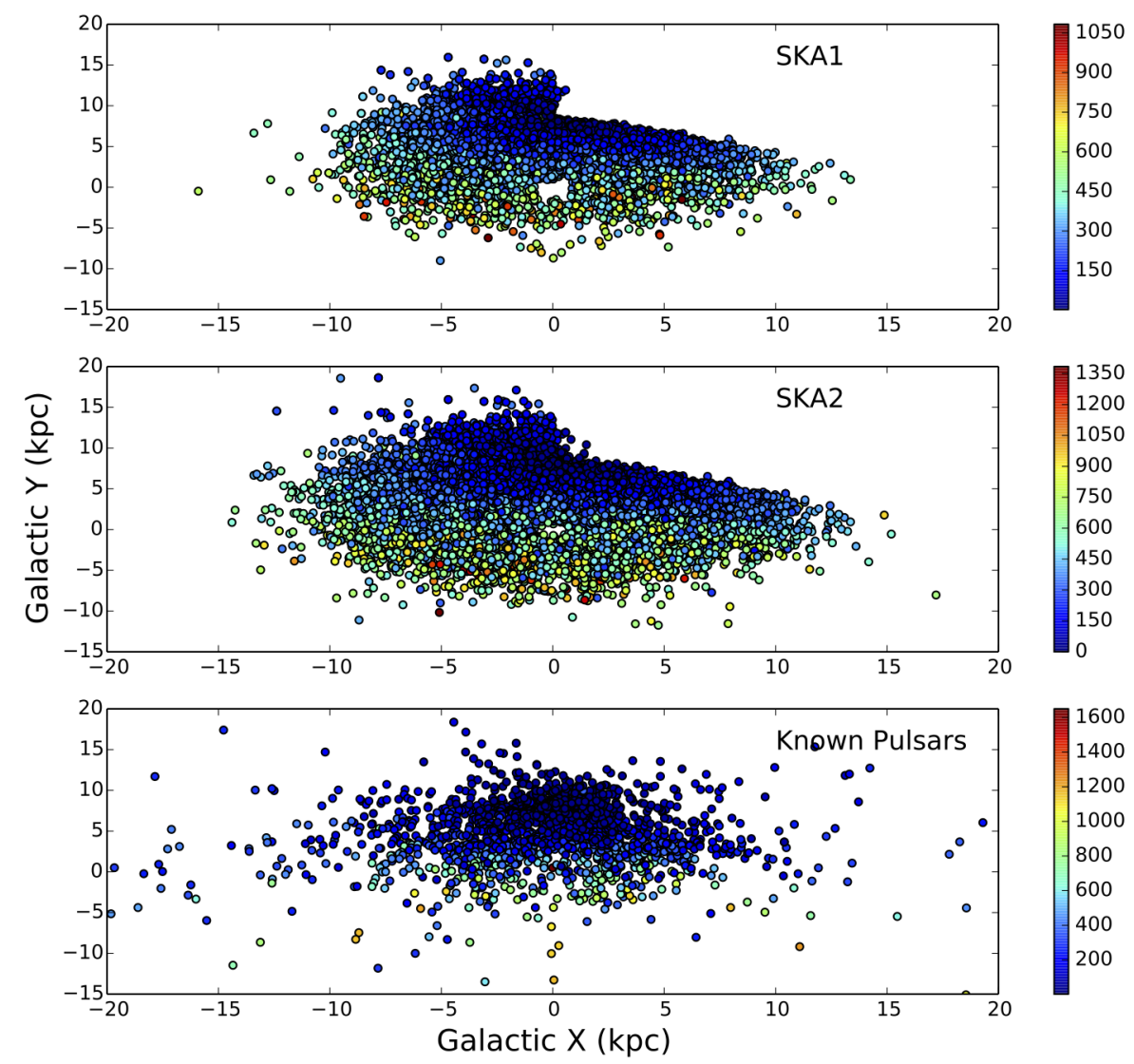

Figure 3: The number of pulsars expected to be found with the SKA and their approximate distribution throughout the Galaxy, projected onto the Galactic plane, compared to the known pulsar distribution. The colour coding indicates the approximate range of dispersion measures of the simulated pulsars that will be discovered. We note the dramatic increase in the number of pulsars discovered in each phase of the SKA, including a much greater sampling of the Galaxy.

of the two surveys, to ensure that the relative sensitivities are understood as required for effective modeling of the population; and secondly because, as we can see from the MSP simulations, it is apparent that in this region of the sky the two telescopes are finding different sources, SKA1-LOW faint nearby objects and SKA1-MID further away objects. This will, therefore, maximize the return on MSPs, the key target of the survey. In this scenario we find that we can detect a total of about 10,000 normal pulsars and as many as 1500 MSPs.

This highlights the importance of having the beam former and pulsar search capabilities available for SKA1-LOW in both phases of the SKA. It also offers up the possibility of an ever-higher pulsar yield, as with the survey being split across both telescopes, the same survey can be achieved in the same time with longer integration times, further enhancing our sensitivity, with the proviso of the highly accelerated binary systems.

We have also examined the yield of a preliminary survey with an early phase SKA1, defined as $50 \%$ the sensitivity of SKA1. For the same integration times as above, a composite survey would detect about 6000 normal pulsars and as many as 700 MSPs. As expected, doubling the integration time to increase the sensitivity results in a higher yield of 7500 normal pulsars and 950 MSPs, but 
at a much higher, and impractical, processing cost (a factor of $\gtrsim 10$ times increase), as per Figure 1.

\subsection{Full SKA Simulations}

We have carried out simulations for the number of pulsars that will be found with SKA2 in both the LOW and MID (DISH) configurations. In the case of LOW we have assumed that there is a four-fold sensitivity increase and that all of that increase can also be applied to the pulsar search application, when compared to SKA1. In the case of MID-DISH the nominal improvement in sensitivity is expected to be about an order of magnitude. However, depending on how those dishes are distributed and the amount of compute resource that is available it may not be possible to utilise all of that increase for pulsar searches. Therefore we consider here two options: the full ten-fold increase can be used and a five-fold increase in sensitivity. With SKA-LOW we find a total of 11,000 pulsars including about 1500 MSPs, while SKA-MID(DISH) will find between 24,000 and 30,000 pulsars, of which between 2400 and 3000 will be MSPs depending on the exact improvement in sensitivity. In some regions of the sky this corresponds to detecting the entire population of pulsars that are beamed in our direction. As with SKA1, LOW and MID are highly complementary with LOW finding the nearby pulsars and MID probing deep into the Galaxy.

Simulations of pulsar surveys with a mid-frequency aperture array centered at $750 \mathrm{MHz}$ with $500 \mathrm{MHz}$ of bandwidth show that such a system would likely be complementary to the LOW and MID surveys already considered. The survey would detect around 27,000 normal pulsars, around 6000 of which would not be detected in either the LOW or MID surveys, and 3000 MSPs, 800 of which would be unique discoveries. Further analysis will be required on computational costs and scientific returns to see how the survey will be distributed over the three antenna types when more detailed specifications are available.

\section{Targeted Searches}

Targeted searches allow for longer integration times and hence better sensitivity than wide-area surveys. This enables characterisation of specific environments in ways unattainable otherwise, and can elucidate evolutionary links between different types of neutron stars and their progenitors.

\subsection{Galactic Centre Pulsars}

The Galactic Centre (GC) is a region of intense interest as pulsars discovered here are excellent tools for measurements of the magnetised ISM in this extreme environment, and could act as unrivaled probes of the space-time surrounding our nearest supermassive black hole candidate, Sgr A* (Liu et al. 2012). Despite strong evidence for a large neutron star population in the GC, e.g. $\gtrsim 1000$ in the central pc around Sgr A* (Wharton et al. 2012; Chennamangalam et al. 2014), and multi-frequency searches for pulsars, the number of detections remains low with just 6 active radio pulsars within $15^{\prime}$ (36 pc) of Sgr A*. This has primarily been explained by extreme scattering of radio waves caused by inhomogeneities in the ionised component of the GC ISM (Cordes \& Lazio 1997; Lazio et al. 1998). Scattering, which causes temporal broadening of pulses, and a corresponding reduction in pulse signal-to-noise ratio $(\mathrm{S} / \mathrm{N})$, can only be mitigated by observing at higher frequencies. Unfortunately, the steep radio spectra of pulsars typically prohibits detection above $2 \mathrm{GHz}$. Finding the optimum balance between the effects of pulse scattering in the GC and 
the intrinsic luminosity of pulsars has been an on-going problem. It is therefore clear that the large increase in sensitivity offered by the proposed collecting areas of SKA1 and the complete SKA will greatly help searches in the GC.

The recent detection of an X-ray and radio loud magnetar just 3" ( $\sim 0.1 \mathrm{pc})$ from Sgr A* has both raised hopes for the possibility of more pulsars in this region, and allowed measurements of the level of pulse scattering, $\tau_{\text {scatt }}$, in this direction (Kennea et al 2013; Mori et al. 2013; Eatough et al. 2013; Shannon \& Johnston 2013; Spitler et al. 2014; Tauris et al. 2015). While the exact nature of scattering toward the GC remains uncertain, these recent measurements suggest that normal slow and some recycled pulsars might be observable in the GC with SKA1-MID bands 3 and 4 ( $\tau_{\text {scatt }} \sim 50 \mathrm{~ms}$ and $7 \mathrm{~ms}$ respectively), whereas MSPs will require band 5 ( $\tau_{\text {scatt }} \sim 4$ ms to $60 \mu \mathrm{s}$ at the bottom and top of band 5 respectively). For a more detailed discussion of the prospects for GC pulsar searches and fundamental physics to be performed therewith we refer the reader to the chapter on observing radio pulsars in the Galactic Centre (Eatough et al. 2015).

\subsection{Extragalactic}

Pulsars beyond the disk of the Milky Way are currently known only in globular clusters and in the Magellanic Clouds, owing to their intrinsic faintness. While single, non-repeating bursts of apparent extragalactic origin have recently been detected (Lorimer et al. 2007; Keane et al. 2012; Thornton et al. 2013; Spitler et al. 2014), their astrophysical source remains unclear. For repetitive, pulsar-like bursts, only much weaker candidates were found. With the SKA, galaxies in the local group are within reach using periodicity searches while giant pulses like those seen from the Crab pulsar can be detected from galaxies out to well beyond a Mpc. What is the importance of detecting pulsars in other Galaxies? The pulsars likely to be detected will be young with high luminosities that can be correlated with catalogs of supernova remnants. This will yield estimates of the star-formation rate and the branching ratio for supernovae to form spin-driven pulsars as opposed to magnetars and black holes. Extragalactic pulsars will also provide information about the magnetoionic media along the line of sight through determination of the dispersion, scattering and rotation measures. Unambiguous study of the intergalactic medium in the local group requires removal of contributions to these measures from the foreground gas in the Galaxy and gas in the host galaxy. The more pulsars detected in a galaxy, the more robust this removal will be. Extragalactic pulsars can be found through blind surveys for both periodic sources and individual giant pulses. Additional successes will follow from targeted surveys of individual supernova remnants in the nearest galaxies. In $\$ 2.1$ we discussed the requirements on sensitivity and FoV. The SKA2MID sensitivity will be applied to targeted searches of, for instance, supernova remnants in nearby galaxies. However, blind surveys over wider fields such as whole galaxies, will only use the core array.

Giant pulses from the Crab pulsar serve as a useful prototype for estimating detection of strong pulses from nearby galaxies. The strongest pulse observed at $0.43 \mathrm{GHz}$ in one hour has $S / N_{\max }=$ $10^{4}$, even with the system noise dominated by the Crab Nebula. For objects in other galaxies, the system noise is dominated by non-nebular contributions, implying that the $\mathrm{S} / \mathrm{N}$ in this case would have increased by a factor of about 300 . We can estimate the maximum distance of detection at a 
specified signal-to-noise ratio, $(S / N)_{\mathrm{det}}$ as:

$$
D_{\max }=\frac{1.6 \mathrm{Mpc}}{\sqrt{(S / N)_{\operatorname{det}} / 5}}\left(\frac{f_{\text {core }} S_{\text {SKA-MID }}}{S_{\text {Arecibo }}}\right)^{1 / 2}=\frac{1.6 \mathrm{Mpc}}{\sqrt{(S / N)_{\operatorname{det}} / 5}}\left(\frac{0.4 \times 1630 \mathrm{~m}^{2} / \mathrm{K}}{1150 \mathrm{~m}^{2} / \mathrm{K}}\right)^{1 / 2}
$$

where $\mathrm{S}_{\mathrm{SKA}-\mathrm{MID}}$ and $S_{\text {Arecibo }}$ are the ratios of effective area over system temperature for SKA-MID and Arecibo, respectively (Table 1 in SKA-TEL-SKO-DD-001); and $f_{\text {core }}$ is the SKA collecting area that can be used for a giant pulse survey. For $f_{\text {core }}=0.4, A_{\mathrm{SKA}} / A_{\text {Arecibo }} \approx 1$, the standard one-per-hour pulse seen at Arecibo could be detected out to $\approx 1.2 \mathrm{Mpc}$.

In conclusion, SKA will enable not only the discovery of most, if not all, pulsars in the Milky Way which are beamed towards Earth, but also allows present-day-survey sensitivities to pulsars in the closest galaxies. With the single-pulse search techniques, it should be possible to detect giant pulses from pulsars as distant as the Virgo Cluster. Studies of the significant numbers of extragalactic pulsars expected to be detectable by the SKA would allow measurements of the intergalactic, as opposed to the interstellar, medium.

\subsection{Globular Clusters \& High Energy Targets}

Targeted searches of globular clusters with SKA1-LOW and SKA1-MID will utilise the vastly improved sensitivity to discover many new exotic systems. Pulsars in globular clusters are subject to a much higher rate of encounters which enables systems to form which would be impossible in the lower density environment of the Milky Way. For a detailed discussion of globular cluster search strategies, and the scienctific applications of these systems, we refer the reader to the chapter on Globular Clusters (Hessels et al. 2015).

Similarly, targeted searches of unidentified sources found by high-energy telescopes (such as the LAT on Fermi) will be employed, as has been done with great success in recent years (Ray et al. 2011). These discoveries are mainly highly energetic young pulsars, and millisecond pulsars, both isolated and in binary systems. The first group of energetic pulsars is opening a new window to the pulsars' radio and high-energy emission mechanisms. The second group, of older neutron stars, is increasing our ability to study neutron star evolution and is adding numbers to the important population of MSPs, that are also required to build a Galaxy-size gravitational wave detector based on these objects (see section 5.2). The SKA will be able to probe high-energy sources that are still unidentified with greater sensitivity, and more generally it will be able to search for unknown radio pulsars in any sources found by telescopes operating across the spectrum. Particularly for very high energy targets the wide FoV of the SKA is also a great advantage. Since in many cases the exact location of the small neutron star within the high-energy emitting region is unknown, current large single-dish telescopes, with their small beams, need to do several pointings in order to overcome these positional uncertainties in the surveys. More on the possibility of finding new pulsars in sources discovered at different wavelengths or in completely different regimes (e.g. gravitational waves and neutrinos) is described in the chapters on multi-messenger pulsar science and on the neutron star population (Antoniadis et al. 2015; Tauris et al. 2015). 


\section{Expected discoveries and their importance}

\subsection{Pulsar-Black Hole Binaries and other binaries for GR tests}

Since early in the conception of the SKA, the detection and study of highly relativistic binary pulsar systems has been one of the Key Science goals (Kramer et al. 2004). Such systems have provided the first strong evidence for the existence of GWs with the double neutron star PSR B1913+16 (Taylor et al. 1979), the most precise tests of Einstein's theory of General Relativity (GR) with the double pulsar PSRs J0737-3039A/B (Kramer et al. 2006), and the best tests of alternative gravity theories with, for example, PSR J0348+0432 (Antoniadis et al. 2013). However, in the collection of these remarkable "gravity labs" the most prized system has so far eluded detection: a pulsar black hole binary (hereafter PSR-BH). The study of a PSR-BH will allow not only precision tests of GR, but should, for the first time, allow the properties of the BH to be measured in a model independent fashion.

The Science prospects from discovery of a PSR-BH system: A PSR-BH system will allow us to probe $\mathrm{BH}$ properties, as well providing stringent tests of theories of gravity in general. If the "No-hair theorem" holds, we expect that BHs are remarkably simple objects described by mass and spin (and perhaps charge) only. Once the mass and spin are known, other properties like the quadrupole moment are pre-determined, so that by measuring all quantities at the same time the nohair theorem can be tested. For the same reason, we do not expect a BH to carry a "scalar charge", in contrast to other compact objects like neutron stars where this is possible. The existence, or not, of a scalar charge results in vastly different behaviours in the orbital motion of a system involving a BH, when predictions of GR are compared with alternatives like tensor-scalar theories. Indeed, it can be argued that a PSR-BH is probably the best foreseeable probe for testing alternative theories of gravity (Damour \& Esposito-Farèse 1996). Moreover, measuring relativistic spin-orbit coupling and frame dragging, will allow us to determine the spin of the $\mathrm{BH}$ with high precision. While the mass of the $\mathrm{BH}$ can be determined with high precision, the (unitless) spin parameter should be measured to be smaller than unity, in order for an event horizon to exist - a fact that can, with the SKA, be easily tested (Kramer et al. 2004). Measuring the effects of classical spin-orbit coupling will also provide the quadrupole moment of the $\mathrm{BH}$, which can then be compared to the predictions based on the mass and the spin. Whether we will be able to measure the spin and quadrupole moment depends also on the mass of the BH. While Liu et al. (2012) have shown that it will be difficult to measure the qudrupole moment for stellar-sized BHs, the BH in the centre of the Milky Way is an ideal target to attempt this experiment (see e.g. Eatough et al. 2015).

The search strategy: Naturally, these exceptional gravity tests will first require the detection of a PSR-BH. As outlined above, a composite survey with SKA-LOW and -MID is proposed to find all pulsars beaming towards us, with significant inroads being made already with SKA1. However, it is fair to say that a PSR-BH might be the most challenging of all the systems in the "pulsar zoo" to detect. In Figure 4 the effectiveness of acceleration searches in the detection of a simulated PSRBH is shown. Panels (a) and (b) show contours of 30\%,60\% and $90 \%$ signal recovery at the orbital phase at which the integration has started ${ }^{5}$. For longer integration times (Panel (a), $\sim 6 \%$ of the

\footnotetext{
${ }^{5}$ Here "signal recovery" is given by the $\mathrm{S} / \mathrm{N}$ achieved in an acceleration search, normalised by the $\mathrm{S} / \mathrm{N}$ for an equivalent search of the same pulsar, but with no orbital motion.
} 

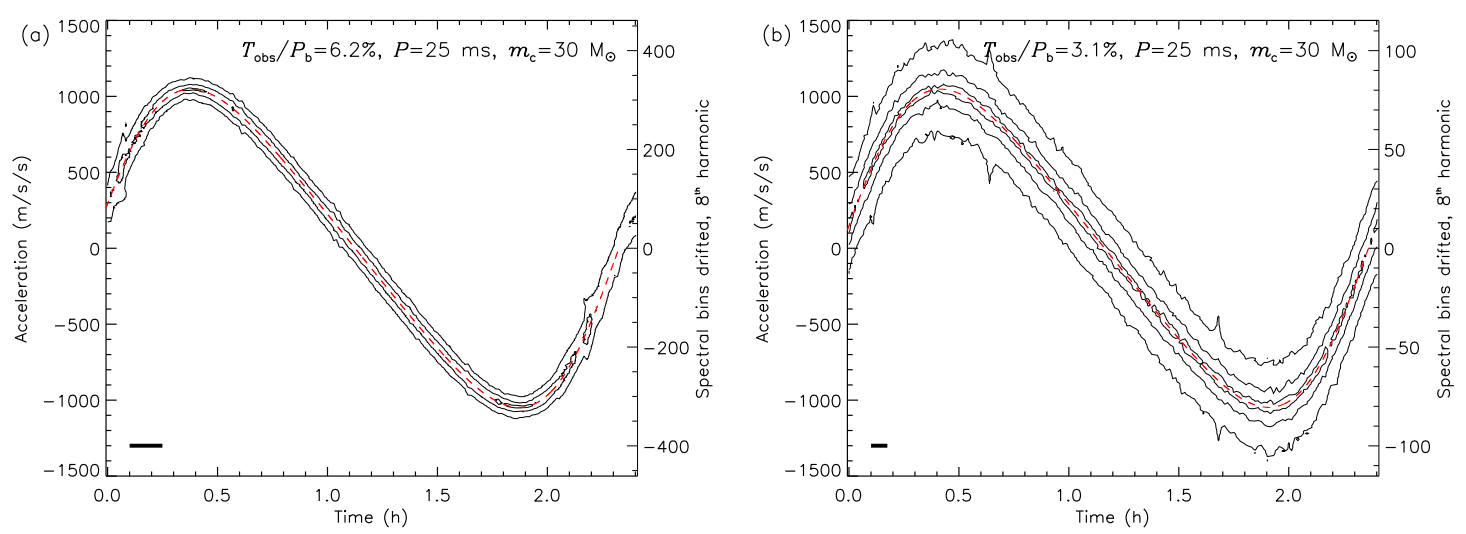

Figure 4: The effectiveness of acceleration searches in the detection of a simulated PSR-BH system. While such a system would be ideal for gravity tests, its detection through blind searches would be extremely challenging. Here we represent a "worst-case scenario", where the pulsar has a short spin period of $25 \mathrm{~ms}$; expected in systems that have formed via exchange interactions in dense stellar environments, or where the pulsar is the first-born object. The black hole mass is at the high end of what is expected for stellar mass black holes $\left(30 \mathrm{M}_{\odot}\right)$, and the orbital period is small $\left(P_{\mathrm{b}}=2.4 \mathrm{hr}\right)$ and has low eccentricity $(e=0.1)$. To worsen matters the system is viewed edge-on, where the deleterious effects of line-of-sight motion are strongest. Contours mark the 30\%, 60\% and 90\% pulsar signal recovery levels for constant acceleration searches of data integrations which are $\sim 6 \%$ and $\sim 3 \%$ of the orbital period (panels (a) and (b) respectively). Because a survey observation can start at any phase in the orbit, the starting point of the acceleration search has been incremented in steps of $50 \mathrm{~s}$ across the entire orbit. The thick black line shows the integration length analyzed. The red dashed line shows the true acceleration value at the mid-point of integration (Liu et al. in prep).

orbital period) full pulsar signal recovery levels are only achieved at a minority of orbital phases where the acceleration is varying least. By reducing the integration time, the amount by which the signal is smeared out (due to higher order effects) in the frequency domain is reduced. Panel (b) shows acceleration searches on integrations with half the duration $(\sim 3 \%$ of orbital period). Although the raw sensitivity would be reduced by a factor of $\sqrt{2}$, the recovery level is now $>90 \%$ over the majority of the orbit. From sensitivity considerations the $60 \%$ contours in Panel (a) are approximately equivalent to the $90 \%$ contours in Panel (b), however there is uneven sensitivity coverage across the entire orbit at $60 \%$ levels in Panel (a). The increased instantaneous sensitivity, and therefore reduced integration time, offered by the SKA will undoubtedly improve the chances of detecting a PSR-BH.

Searches with SKA1 and SKA2 are expected to be performed in real-time (or pseudo realtime) because of data storage constraints. In searches for PSR-BH systems, where extreme levels of orbital acceleration might be observed (see Figure 4 where the line of sight acceleration can be greater than $1000 \mathrm{~m} \mathrm{~s}^{-2}$ ), even one-dimensional acceleration searches constitute an extreme data processing task. The degree of computational requirements of time-domain pulsar acceleration search algorithms has a strong dependence on the integration time $\left(\propto T^{3}\right)$. As shown in Figure 4, acceleration analyses of half length integrations can result in an equivalent sensitivity to extreme PSR-BH as in longer integrations, but with 8 times less computational expense. Of course, it is only because of the superb sensitivity of the SKA that integration times will be able to be kept to a minimum. In addition, the reduced integration length also implies that more computational effort can be spent searching a wider parameter space. 


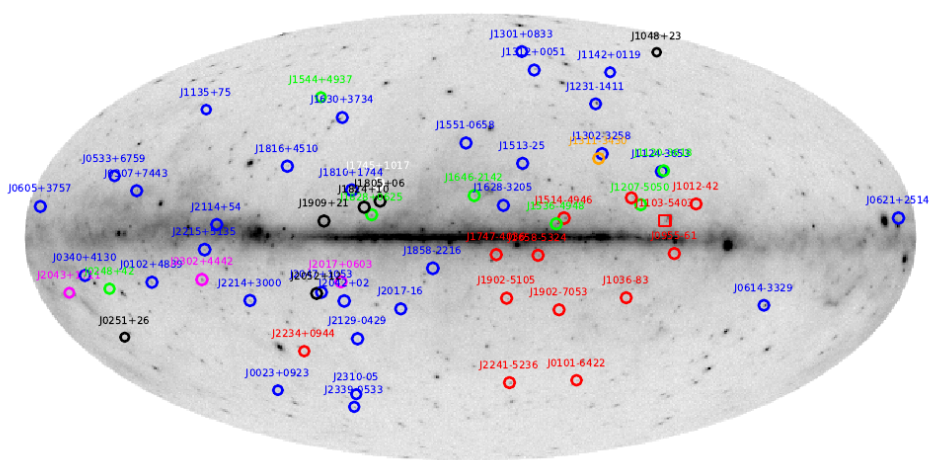

Figure 5: The initial $\sim 60$ newly discovered radio MSPs detected via pointed radio searches towards Fermi associated $\gamma$-ray sources. The nearly isotropic distribution of the relatively nearby $(\sim 1-3 \mathrm{kpc})$ systems is obvious. The colours indicate the discovery telescope: blue is the GBT, red is Parkes, black is Arecibo, green is the GMRT, magenta is Nançay, and white is Effelsberg. SKA surveys should find thousands of new MSPs, perhaps 5-10\% of which will be suitable for high-precision timing work. Searches, especially with SKA1, of pulsar-like Fermi associated sources for new MSPs will provide excellent early-science opportunities for the SKA. Figure courtesy Paul Ray, NRL.

\subsection{MSPs for PTAs and GW searches}

Millisecond pulsars live $10^{2}-10^{3}$ times longer than $\sim 1$-sec pulsars and have substantially larger scale heights in the Galaxy. For these reasons, the majority of the $\sim 200$ currently known Galactic MSPs are local objects within $\sim 1-2 \mathrm{kpc}$ from the Sun, and as such, are distributed nearly isotropically on the sky (see Figure 5). Recent population studies (Grégoire \& Knödlseder 2013; Lorimer 2013; Levin et al. 2013) suggest that the Galaxy holds about 30,000 detectable MSPs in total, thousands of which will be within reach of SKA pulsar surveys. The MSPs are undoubtedly some of the most difficult pulsars to detect, due to their rapid spin rates (demanding fast sampling and high frequency resolution) and their typical binary nature. However, their high rotational stability and related timing precision makes these searches eminently worthwhile, as they can be used for some of our most important physics experiments, such as the direct detection of nano-Hzfrequency GWs (Manchester 2013).

Pulsar timing arrays require the very best MSPs, which are selected based on their flux density, the shapes of their radio pulses (narrow features are better), their timing stability (unknown until measured), and their distribution across the sky (a nearly isotropic distribution is close to ideal for the detection of a stochastic GW background). SKA all-sky surveys will find thousands of new MSPs, but only a relatively small fraction — perhaps 5-10\% — will be of sufficient quality to include in an SKA-based PTA (e.g. Figure 6). Finding these rare pulsars is crucial as recent work has shown that GW sensitivities are directly proportional to the number of 'good' pulsars being timed (Siemens et al. 2013). In addition, these ultra-stable MSPs will provide spectacular 'secondary' science such as high-precision pulsar masses which will constrain the Equation of State of nuclear matter (Demorest et al 2010).

\subsection{Testing the neutron star equation of state}

Together with precise mass determinations in binary systems (see e.g. Demorest et al 2010; Antoniadis et al. 2013), pulsar spin rates are among the most accurately known observables which 


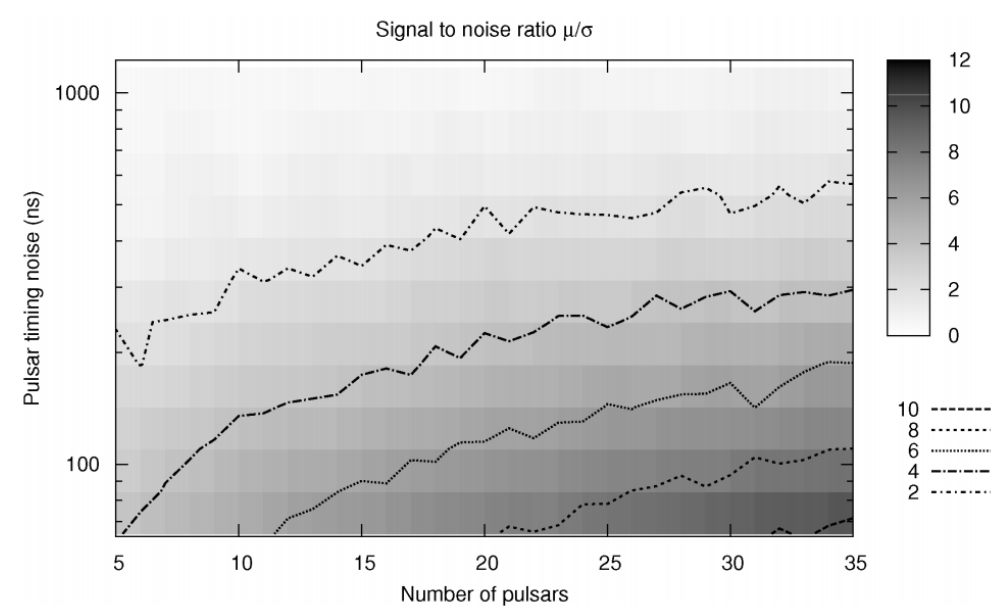

Figure 6: Density plot (grey scale) and contours of the signal-to-noise ratio for GW detection for different realisations of pulsar timing residuals, as a function of pulsar timing noise and the number of pulsars timed. Here, we have assumed 100 data points per pulsars, approximately evenly distributed over a period of 7.5 years and a simulated GW background with amplitude of $10^{15} \mathrm{yr}^{1 / 2}$ (van Haasteren et al. 2009).

could constrain the neutron star EoS. Pulsars can only be spun up to a limiting period, $P_{\text {sh }}$, below which the star, due to centrifugal forces, becomes unstable to mass shedding at its equator. The binary MSP J1748-2446ad (Hessels et al. 2006), is the neutron star with the shortest known rotational period, $P_{\min }=1.396 \mathrm{~ms}^{6}$, implying that the radius, if it were a canonical $1.4 \mathrm{M}_{\odot}$ neutron star, should be smaller than $\sim 15 \mathrm{~km}$ (Hessels et al. 2006). Irrespective of the proposed EoSs, the period $P_{\min }$ of J1748-2446ad must be longer than $P_{\mathrm{sh}}$. The value of $P_{\mathrm{sh}}$ strongly depends on the EoS (Phinney \& Kulkarni 1994; Stergioulas \& Friedman 1995), with the observed $P_{\min }$ very close to the mass-shedding limit for the most 'stiff' EoSs (see Figure 7). However, the re-acceleration of a neutron star with an initial mass of $1.4 \mathrm{M}_{\odot}$ could in principle proceed down to limiting spin periods as fast as $0.6 \mathrm{~ms}$ for most 'soft' EoSs (Cook et al. 1994). As a consequence, even the discovery of one sub-ms pulsar, with a rotational frequency well above $1 \mathrm{kHz}$, would lead to the rejection of a wide class of EoSs and provide crucial information about the behaviour of matter at supra-nuclear densities.

Although few recent pulsar surveys could have detected sub-millisecond radio pulsations, a strong bias exists against detecting fast spinning MSPs as a result of the observed preference of these neutron stars to be hosted in eclipsing binaries ( 9 cases out of a total of 11 binary MSPs with $P<2$ ms, including PSR J1748-2446ad). In these systems matter released by the companion engulfs the system and obscures the radio pulsations for a large fraction of the orbital period and, occasionally, for the entire orbit, particularly at lower radio frequencies. Also, a pulsar like $\mathrm{J} 1748-2446 \mathrm{ad}$ is too faint (about $80 \mu \mathrm{Jy}$ at $1.95 \mathrm{GHz}$ ) to be detectable by most of the past and/or ongoing large scale surveys. Most of these difficulties will be overcome, or strongly mitigated, by a deep all-sky survey at a frequency of about $2 \mathrm{GHz}$ with SKA1-MID. Provided sub-ms pulsars exist, and they mostly reside in eclipsing binaries, this experiment will provide an unprecedented opportunity to uncover these objects.

\footnotetext{
${ }^{6}$ An 0.89-ms period for the X-ray source XTE J1739-285 (Kaaret et al. 2007) still awaits confirmation.
} 


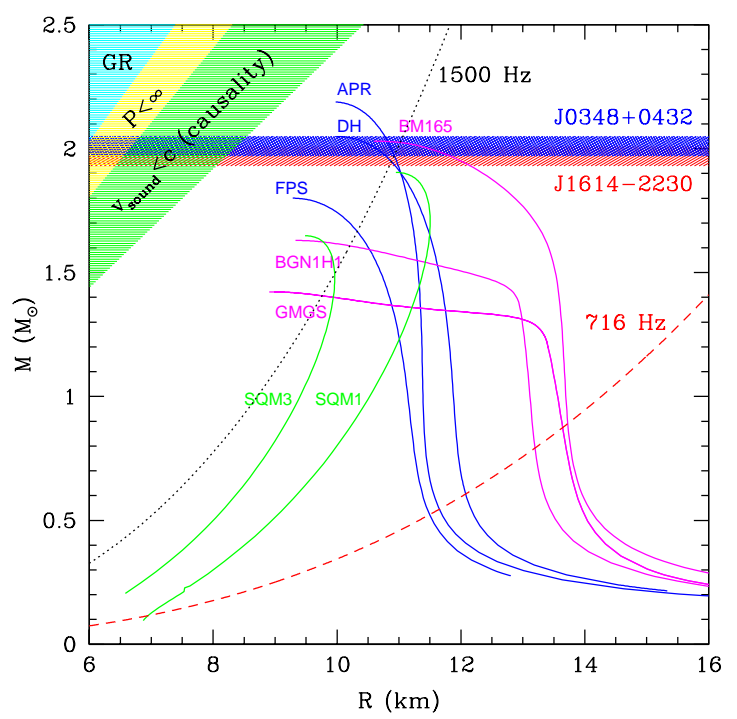

Figure 7: Different equations of state are shown, with regions in the neutron star mass versus radius parameter space which are ruled out or as yet allowed highlighted. Figure adapted from Demorest et al (2010). For further details we refer the reader to the chapter on probing the NS interior and the cold dense matter equation of state (Watts et al. 2015).

\section{Importance of follow-up timing and strategies}

When new pulsars are discovered their rotational, astrometric and binary properties (in case of a companion), need to be determined in order to be able to acquire a stable timing model that can be used to predict the arrival times of the pulses. It is this which enables the many high precision measurements possible using pulsars. The first approximation of a timing model requires very regular observations, starting from daily monitoring to gradually increased spacing between observations. In order to separate the position of the pulsar from the basic spin parameters, at least half a year of timing is necessary (although positional determination using VLBI methods, where possible, can remove this requirement). Pulsars in binary orbits need a high-cadence sampling of the binary orbit to measure the first-order binary parameters. In case of relativistic binaries, the post-Keplerian parameters can usually only be measured after multiple years of timing.

For all pulsars, multi-band monitoring is required to mitigate ISM effects on the signal. In general, lower frequencies are better for measuring dispersion measure variations and scattering delays, and therefore we expect most pulsars will require monitoring at the lower bands of SKAMID. In addition, SKA-LOW can also be used for follow-up timing for the relatively brighter and/or more nearby pulsars. However, in some cases higher frequencies (around $3 \mathrm{GHz}$ ) will be essential to get a better coverage of binary orbits. As described above, the fastest-spinning pulsars are likely to be found in eclipsing binaries. Due to the fact that the effect on the higher-frequency part of the signal is smaller, the pulses can be detected for a larger fraction of the orbit at those frequencies and makes determining and monitoring the binary parameters possible or easier.

We aim for regular follow-up for all known and newly discovered pulsars visible in the SKA sky. Even the slow pulsars need to be covered in the SKA timing programme on a regular basis. The 45 -year timing programme at Jodrell Bank, where up to 800 pulsars have been observed, has 
led to unexpected results on various timescales for all kinds of pulsars (Lyne et al. 2010; Espinoza et al. 2011; Lyne et al. 2013) showing that different types of pulsars require different strategies in their follow-up and long-term timing observations.

Pulsars that show timing noise or rotational irregularities: These are typically young pulsars, or high-magnetic field pulsars that show timing irregularities, mode changes on various timescales or glitches. The required cadence for observing depends on the relevant variability time scales and varies from once a week to once a month. Many pulsars exhibit glitches, sudden (probably instantaneous) increases in the rotation rate of a pulsar that happen on different timescales and on an irregular basis (Espinoza et al. 2011). They are thought to be the result of changes in the interior of the neutron star where angular momentum is transferred from the interior to the crust. Therefore closely monitoring the recovery in the spin parameters of the pulsar after a glitch can provide us with information of the glitch mechanism itself, and the equation-of-state inside the neutron star (for more details see Watts et al. 2015). Intermittent and mode-changing pulsars: these sources show differences in spin-down rates on various timescales (Kramer et al. 2006; Lyne et al. 2010). An observing cadence matching the time scale of the variability is required. Rotational RAdio Transients (RRATs): These are neutron stars that only emit detectable single pulses (McLaughlin et al. 2006; Keane \& McLaughlin 2011) on a very irregular basis and therefore require more integrated observing time and/or a higher cadence per source to get to a stable timing solution.

Binary MSPs: observations at least once a month, and occasional high-cadence (or full-orbit, when orbital periods are less than about a day) orbital sampling are required to measure the orbital parameters. GR tests need both dense orbital coverage to measure the binary parameters accurately, as well as a long-term timing programme to detect and monitor secular changes in binary orbits and astrometric parameters, and to disentangle those. Exotic binary MSPs: the SKA promises to uncover many exotic systems, e.g. when PSR-BH systems are found we might expect that the postKeplerian effects on the orbit could become more complex than in the currently known systems that are used for GR tests. Second, or higher order, post-Newtonian effects would then become important requiring long-term and high-cadence observations. In this scenario it is necessary to obtain a high-cadence coverage of the PSR-BH binary orbit (or full-orbit observations, depending on the orbital period) as well as daily monitoring of the system to ensure coherence is maintained. It is likely that other exotic systems like triple systems will be found, especially in globular cluster searches, as multi-body systems are more likely to be found there. It has been shown (Ransom et al. 2014) that daily monitoring is required to maintain coherence for those systems.

PTA pulsars: one of the main goals of long-term timing is to directly detect low-frequency GWs using a pulsar timing array. A set of stable MSPs is used as a Galaxy-scale GW detector which depends on having long-term timing observations for pulsars distributed across the sky. There are different classes of GWs which may each require a slightly different observing strategy (for more information, see the chapter on "Gravitational wave astronomy with the SKA", Janssen et al. 2015). Overall, given the large number of MSPs that the SKA will discover (see above), for GW work the requirements are: (1) observe as many MSPs as possible on a regular basis with multi-frequency coverage; and (2) identify the most stable pulsars by long-term monitoring and determining the red-noise contamination in their timing residuals. As most PTA pulsars are in binaries, they have the same requirements as those listed above for the binary pulsars.

Follow-up pulsar timing also supports multi-wavelength observations by providing precise 
ephemerides. Over a hundred pulsars are now known to emit pulses in the $X$-ray and/or $\gamma$-ray regimes. To fold their photons correctly, in many cases the $\gamma$-ray and sometimes $\mathrm{X}$-ray communities are dependent on up-to-date timing ephemerides based on radio observations. Also, multiwavelength counterpart observations can be used to independently constrain distances, and study the pulsar emission mechanism. Thus the short-term follow-up timing of all newly discovered sources requires a short-spacing campaign to make an initial coherent timing solution. Depending on the parameters and the complexity of the source (timing irregularities, binary parameters, etc) the longer-term timing cadence can be expected to be somewhere between daily and monthly, with full-orbit campaigns on a regular basis for the most important or complex systems.

In this chapter, we have demonstrated that significant achievements can be made in pulsar searching in the early science phase of SKAl with a highly concentrated core. In contrast, in order to achieve the highest precision in pulsar timing, the gain in using SKA2 is enormous when compared to timing observations enabled with SKA1. It is this gain in timing precision for selected KSP objects which will ultimately require the sensitivity of SKA2.

\section{References}

Antoniadis, I., Freire, P. C. C., Wex, N., et al., 2013 Science, 340, 448

Bates, S. D., Lorimer, D. R., Verbiest, J. P. W. 2013, MNRAS, 431, 1352

Bates, S. D., Lorimer, D. R., Rane, A. \& Swiggum, J., 2014, MNRAS, 439, 2893

Chennamangalam, J, Lorimer, D. R., 2014, MNRAS, 440, L86-L90

Cordes, J. M., \& Lazio, J. T. W. 1997, ApJ, 475, 557

Cook, G. B., Shapiro, S. L. \& Teukolsky, S. A. 1994, ApJ, 424, 823

Damour, T. \& Esposito-Farèse, G., 1996, PhysRevD, 54, 1474

Demorest, P.B., Pennucci, T., Ransom, S.M., Roberts, M.S.E. \& Hessels, J.W.T., 2010, Nature, 467, 1081

Eatough, R. P., Molkenthin, N., Kramer, M. et al., 2010, MNRAS, 407, 2443

Eatough, R. P., Falcke, H., Karuppusamy, R. et al. 2013, Nature, 501, 391E

Eatough, R. P., Lazio, J. T. W., Casanellas, J. et al., 2015, "Observing Radio Pulsars in the Galactic Centre with the Square Kilometre Array", in proc. Advancing Astrophysics with the Square Kilometre Array, PoS(AASKA14)045

Espinoza, C. M., Lyne A. G., Stappers, B. W. \& Kramer, M., 2011, MNRAS, 414, 1679

Grégoire T \& Knödlseder J., 2013, A\&A, 554, A62

Antoniadis. J, Guillemot, L., Possenti, A., et al., 2015, "Multi-wavelength, Multi-Messenger Pulsar Science in the SKA Era", in proc. Advancing Astrophysics with the Square Kilometre Array, PoS(AASKA14) 157

van Haasteren, R., Levin, Y., McDonald, P. \& Lu, T., 2009, MNRAS, 395, 1005

Hessels, J. W. T., Ransom, S. M., Stairs, I. H. et al., 2006, Science, 311, 1901 730, L36

Hessels, J. W. T., Possenti, A., Bailes, M. et al., 2015, "Pulsars in Globular Clusters with the SKA", in proc. Advancing Astrophysics with the Square Kilometre Array, PoS(AASKA14)047 
Janssen, G. H., Hobbs, G., McLaughlin, M. A. et al., 2015, "Gravitational Wave Astronomy with the SKA", in proc. Advancing Astrophysics with the Square Kilometre Array, PoS(AASKA14)037

Kaaret, P., Prieskorn, Z., in 't Zand, J. J. et al., 2007, ApJ, 657, L97

Kennea, J. A., Burrows, D. N., Kouveliotou, C. et al., 2013, ApJ, 770L, 24

Keane, E. F. \& McLaughlin, M. A., 2011, BASI, 39, 333

Keane, E.F., Stappers, B.W., Kramer, M. \& Lyne, A.G., 2012, MNRAS, 425, L71

Kramer, M., Backer, D. C., Cordes, J. M. et al., 2004, New Astronomy Reviews, 48, 993

Kramer, M., Stairs, I. H., Manchester, R. N. et al., 2006, Science, 314, 97

Lee, K. J., Stovall, K., Jenet, F. A. et al., 2013, MNRAS, 433, 688

Lazio, T. J. W. \& Cordes, J. M., 1998, ApJ, 505, 715

van Leeuwen, J. \& Stappers, B. W., 2010, A\&A, 509, 7

Levin, L., Bailes, M., Barsdell, B. R. et al. 2013, MNRAS, 434, 1387

Liu, K., Wex, N., Kramer, M. \& Cordes, J. M., 2012, ApJ, L747, 1

Lorimer, D. R. \& Kramer, M., 2005, Handbook of Pulsar Astronomy, CUP.

Lorimer, D. R., 2013, IAU, 291, 237

Lorimer, D.R., Bailes, M., McLaughlin, M.A., Narkevic, D.J. \& Crawford, F. 2007, Science, 318

Lyon, R., Brooke, J. M., Knowles, J. D. \& Stappers, B. W., 2013, IEEE International Conference on Systems, Man, and Cybernetics.

Lyne, A. G., Hobbs, G., Kramer, M. \& Stairs I. H., 2010, Science, 329, 408

Lyne, A. G., Graham-Smith, F., Weltevrede, P. et al., 2013, Science, 342, 598

Mori, K., Gotthelf, E. V., Zhang, S. et al., 2013, ApJ, 770L, 23M

Manchester, R.N., Hobbs, G.B., Teoh, A. \& Hobbs, M. 2005, VRODC, 7245

Manchester R. N., IPTA, 2013, CQG 30: 224010

Macquart, J.-P., Keane, E. F., Grainge, K. et al. 2015, "Fast Transients at Cosmological Distances with the SKA", in proc. Advancing Astrophysics with the Square Kilometre Array, PoS(AASKA14)055

McLaughlin M. A., Lyne, A. G., Lorimer, D. R. et al., 2006, Nature, 439, 817

Morello, V., Barr, E. D., Bailes, M. et al., 2014, MNRAS, 443, 1651

Phinney, E. S., \& Kulkarni, S. R. 1994, ARAA, 32, 591

Ray, P. S., Abdo, A. A., Parent, D. et al. 2011, Fermi Symposium proceedings - eConf C110509

Ransom, S. M., Stairs, I. H., Archibald, A. M. et al. 2014, Nature, 505, 520.

Siemens X., Ellis J., Jenet F. \& Romano J. D. 2013, CQG 30: 224015.

Shannon, R. M. \& Johnston, S., MNRAS, 2013, 435L, 29

Smits, R., Kramer, M. \& Stappers, B. W., 2009, A\&A, 493, 1161

Spitler, L. G., Lee, K. J., Eatough, R. P. et al. 2014, ApJ, 780L, 3

Stergioulas, N., \& Friedman, J. 1995, ApJ, 444, 306

Tauris, T. M., Kaspi, V. M. Breton, R. P. et al., 2015, "Understanding the Neutron Star Population with the SKA", in proc. Advancing Astrophysics with the Square Kilometre Array, PoS(AASKA14)039

Taylor, J. H., Fowler, L. A. \& McCulloch, P. M., 1979, Nature, 277, 437

Thornton, D., Stappers, B., Bailes, M. et al. 2013, Science, 341

Watts, A. L, Xu, R., Espinoza, C. M. et al. 2015, "Probing the neutron star interior and the Equation 
of State of cold dense matter with the SKA", in proc. Advancing Astrophysics with the Square Kilometre Array, PoS(AASKA14)043

Wharton, R. S., Chatterjee, S., Cordes, J. M., Deneva, J. S. \& Lazio, T. J. W., 2012, ApJ, 753, 108 Zhu, X.-J., Hobbs, G., Wen, L. et al., 2014, ApJ, 781, 117 